This item was submitted to Loughborough's Research Repository by the author.

Items in Figshare are protected by copyright, with all rights reserved, unless otherwise indicated.

\title{
Investigation of wave stripping models on a generic wing-mirror using a coupled level-set volume of fluid simulation
}

PLEASE CITE THE PUBLISHED VERSION

https://doi.org/10.4271/2020-01-0682

PUBLISHER

SAE

VERSION

AM (Accepted Manuscript)

\section{PUBLISHER STATEMENT}

This paper was accepted for publication in the journal SAE Technical Papers and the definitive published version is available at https://doi.org/10.4271/2020-01-0682

\section{LICENCE}

CC BY-NC-ND 4.0

\section{REPOSITORY RECORD}

Skarysz, Maciej, Andrew Garmory, J Escobar, J Jilesen, and A Gaylard. 2020. "Investigation of Wave Stripping Models on a Generic Wing-mirror Using a Coupled Level-set Volume of Fluid Simulation". Loughborough University. https://hdl.handle.net/2134/12278090.v1. 


\title{
Investigation of wave stripping models on a generic wing-mirror using a Coupled Level-set Volume of Fluid simulation
}

\author{
Author, co-author (Do NOT enter this information. It will be pulled from participant tab in \\ MyTechZone) \\ Affiliation (Do NOT enter this information. It will be pulled from participant tab in MyTechZone)
}

\begin{abstract}
Predicting Exterior Water Management is important for developing vehicles that meet customer expectations in adverse weather. Fluid film methods, with Lagrangian tracking, can provide spray and surface water simulations for complex vehicle geometries in on-road conditions. To cope with this complexity and provide practical engineering simulations, such methods rely on empirical sub-models to predict phenomena such as the film stripping from the surface. Experimental data to develop and validate such models is difficult to obtain therefore here a high-fidelity Coupled Level-set Volume of Fluid (CLSVOF) simulation is carried out. CLSVOF resolves the interface of the liquid in three dimensions; allowing direct simulation of film behaviour and interaction with the surrounding air. This is used to simulate a simplified wing-mirror, with air flow, on which water is introduced. The film shows very different behaviour on the in-board section, where a film is developed which eventually breaks to rivulets, and the end of the mirror, where the water is rapidly stripped off the surface due to the higher shear stress from the air. The same case is simulated using a fluid film method, which shows that a simple film stripping model based on film height is not capable of predicting the different regimes observed with CLSVOF.

However, a model based on wave stripping due to Kelvin-Helmholtz instability is seen to give good agreement, as was a model based on local film velocity, surface curvature and body force. As well as informing the development of a film stripping model, this also illustrates how a high-fidelity simulation can be used as a tool for developing practical engineering software.
\end{abstract}

\section{Introduction}

Automotive Exterior Water Management (EWM) concerns the control of rainwater flowing over external vehicle surfaces. This is important from the safety perspective as, for example, rainwater can obscure the driver's vision when water flows from the front windshield to the side glass over the A-pillar, or water strips of the wing mirror. Furthermore, increasing usage of onboard cameras and optical sensors requires control of the flow of water over surfaces, as contamination of such sensors will impact their performance and as a result performance of the whole system will suffer. These factors become increasingly important when self-driving systems are introduced, therefore it is desired to keep the surfaces of sensors unobscured in any weather conditions. Moreover, water and contamination on front lights can reduce both the vision of drivers and the visibility of vehicles. At the rear, as water and contaminants accumulate on the rear screen, lights and license plate, they compromise both vision and visibility. When a car is stationary drips of rainwater can fall inside during opening of doors or boot, therefore seals and channels must be designed to prevent this. Finally, aesthetics play an important role for car manufacturers, as efforts of designers can be ruined when the surface of the vehicle is extensively contaminated. Accurate simulation of these EWM processes can allow problems to be identified and rectified at an early design stage. For further reviews of the EWM field and current numerical simulation approaches see $[1,2]$.

From a physical perspective, three different stages occur when practical EWM situations are considered. These are droplet impacts, surface flow, and re-entertainment back to the air-flow field. Different time and length-scales are associated with these phenomena, thus when studied numerically they require special attention. The numerical method widely used by automotive manufacturers is Lagrangian particle tracking for airborne droplets and a $2 \mathrm{D}$ thin film method for surface flow $[3,4,5,6]$. The main advantage of these methods is computational efficiency - it is feasible to study entire vehicle geometry exposed to rain in a computational domain. The high efficiency is obtained by making certain assumptions, such as that the airborne droplets are spherical and can be considered as points, or the surface film is two-dimensional, and its thickness is of order $\mathrm{O}(0.1 \mathrm{~mm})$. The latter assumption can be broken when geometries with high curvature are considered, such as drainage channels. The full interaction of the liquid film and air flow cannot be directly modelled with a thin film model. In this approach droplet impact and re-entertainment cannot be simulated directly, thus these phenomena require additional submodels which will capture missing physics. The submodels must be derived from experimental, numerical or theoretical analysis and are valid for certain flow regimes. Relevant and reproducible experimental data can be difficult to obtain for EWM scenarios and theoretical analysis can be hard to apply to practical situations. Therefore, the use of high-fidelity numerical simulations to derive and validate submodels for use in practical simulations is attractive.

One such numerical approach is to use an interface resolving approach, which does not make assumptions about the liquid phase, as is done in Lagrangian and thin film methods, but fully resolves the 3D shape of the interface between air and fluid phases. These highfidelity results are obtained at high computational cost, as the method requires mesh spacing be below the characteristic length of flow features of interest, which in cases of EWM can be as low as $0.1 \mathrm{~mm}$, or lower when a splash is considered. Therefore these methods are 
usually employed for simple, isolated cases such as a droplet or rivulet flowing into a drainage channel for EWM applications [7], or single droplet impacts such as in work of [8] who studied oblique impacts of droplets of diameters below $200 \mu \mathrm{m}$ for aerospace icing purposes.

This paper uses a high fidelity Coupled Level-Set Volume of Fluid (CLSVOF) method, developed in previous work for EWM ([7, 9, 10]), to simulate stripping of water from the surface of a representative geometry. The same case is then simulated using a 2D fluid film method which employs empirical submodels for reentrainment of the surface water into the air due to film stripping. In this way the performance of the film stripping models can be assessed by comparison with the CLSVOF method. The use of Adaptive Mesh Refinement (AMR) allows the CLSVOF simulations to resolve details of wave formation and film stripping without the need for prohibitively high total mesh sizes. The stripping models assessed are one based on local film height, a more complex model based on wave stripping due to Kelvin-Helmholtz instability and one based on local film velocity, surface curvature and body force. The geometry used in this paper is a simplified wing mirror, which was previously been studied in [11] and [12] in single phase simulations. The wing mirror is additionally considered here with a drainage channel placed close to the edge. This geometry is directly relevant to simulations of re-entraiment of spray from wing mirrors but also gives several different flow regimes in which a film stripping model may need to perform. The results can therefore inform the use of film stripping models more generally in EWM. The paper also demonstrates how one-off high-fidelity simulations can be used as a tool for developing practical engineering software.

\section{Methodology}

In this study two CFD approaches were used for multiphase simulations, these were a Lagrangian particle tracking with thin film model as a part of a commercial Lattice Boltzmann solver ${ }^{\mathbf{T M}^{\mathrm{TM}}}$, and the Coupled Level Set and Volume of Fluid method of $[9,10]$ with Adaptive Mesh Refinement implemented in the open source software OpenFOAM [13].

\section{Particle tracking and thin film model}

PowerFLOW ${ }^{\mathrm{TM}}$ is a commercial numerical solver based on the Lattice-Boltzman method (LBM) $[14,15]$. LBM differs from traditional finite volume methods (FVM) in the sense that it is formulated in terms of molecular motions instead of the continuous systems used in the FVM. In general, a system can be expressed in terms of particle motions at the molecular scale. The LBM describes the system behavior in terms of a distribution function which is defined as the number density of particles. This distribution function provides information about the position and velocity of the particles. Correct discretization of the distribution function in space and the velocity domains guarantees the recovery of the Navier-Stokes equations. Kotapati et al. [16] provides a detailed explanation of LBM as used in this study for the solution of the flow field. Turbulence is accounted for in PowerFLOWTM solver by using an approach that can be described as Very Large Eddy Simulation (VLES) [14]. In this approach the large turbulent structures are solved directly on the computational grid, whereas small structures are modeled. Direct solution of the large turbulent structures captures the anisotropy that emerges in the flow field, especially where flow separation occurs. On the other hand, the more uniform (universal) turbulence scale was modeled using the RNG k-epsilon formulation.
LBM is well suited for performing transient simulations because it is, by definition, unsteady and has very low numerical diffusion.

Within the solver, a two-way coupled Lagrangian particle tracking algorithm is integrated, which is used for simulating airborne droplets, such as rain droplets or tyre spray droplets. The equation of motion for particles neglects all forces except gravity and drag force, which is calculated by the assumption of spherical shape and drag coefficient as a function of slip Reynolds number - Schiller Naumann correlation for small spheres. This correlation remains valid for small Weber numbers, below which the droplet remains spherical and does not deform or break-up. Above $\mathrm{We}=10$ a break-up model can be used, which splits droplet into smaller particles.

Once a particle impacts solid boundary it is transferred into the liquid thin film description. This can also include a splash model, which transfers some of the particle mass back into the air flowfield [17] as Lagrangian drops. The motion of the film is described by a momentum equation $(\mathrm{Eq}(1))$ which accounts for the shear stresses acting on both the air-liquid interface and the liquid-solid interface and gravity acting as a volume force:

$$
\rho h \frac{D \boldsymbol{u}}{D t}=-2 \mu \frac{\boldsymbol{u}}{h}+\tau_{\text {air }}\left(\boldsymbol{u}_{*}, \boldsymbol{n}\right)+\rho(\boldsymbol{g}-(\boldsymbol{g} \cdot \boldsymbol{n})) h
$$

where $\rho, \mu$, and $\mathrm{h}$ are the film density, viscosity and thickness respectively, $\boldsymbol{u}$ is $2 \mathrm{D}$ film velocity. Gravity and surface normal are denoted as $\boldsymbol{g}$ and $\boldsymbol{n}$. The shear stress between the film and the moving air, $\tau_{\text {air }}$, is a function of near wall air velocity, $\boldsymbol{u}_{*}$, and is provided by the flow solver. The method is computationally efficient, however this is at the expense of using an assumption of twodimensionality of the film. It is assumed that velocity in the fluid has a linear profile over height, which is correct for small thickness $\mathrm{O}(0.1 \mathrm{~mm})[18]$.

For film re-entrainment, either by edge stripping or by wave stripping, the method requires an empirical or theoretical model which emits particles back to air flowfield from the film, based on some specific criteria. In this paper three models are tested: A simple model is used which emits particles if the specified film thickness is exceeded. A second model, based on Kelvin-Helmholtz instability, is tested as a post-processing step. Finally, a model based on local film velocity, surface curvature and body force is tested. In general, mesh spacing in this method is larger than the liquid structures modelled. By contrast, to fully resolve the 3D shape of the interface in Eulerian methods, and model stripping directly, the mesh needs to be finer than droplets diameter as is discussed next.

\section{Coupled Level Set and Volume of Fluid with Adaptive Mesh Refinement}

A Coupled Level-Set Volume of Fluid method has been developed in OpenFOAM for EWM in [9, 10] and applied in [7, 19]. The reader is referred to these references for details of the method, but in brief transport equations are solved for Level-Set (signed distance from the interface) and Volume of Fluid (fraction of liquid in cell). These are then used to 'reconstruct' the gas-liquid interface. This allows for a combination of sharp interface resolution and mass conservation. For accurate simulation of film stripping the mesh must resolve the development of surface waves and subsequent stripping. This requires a fine mesh resolution, which would be impractical to 
maintain over all the region of interest. Therefore Adaptive Mesh Refinement (AMR) is employed so that the mesh is only resolved where required. As the gas-liquid interface is resolved in detail it is also necessary to resolve turbulent motion and so a Large Eddy Simulation (LES) turbulence modelling approach is used.

The AMR used in this study was originally implemented as a part of OpenFOAM CFD library for general hexahedral cells and was added to the implementation of CLSVOF solver used here. A hexahedral unstructured mesh is used, and refinement is based on octree split of cells, such that a cell after refinement is split to 8 new hexahedral cells. The purpose of this is to capture interface of high curvature and be able to resolve thin filaments. Two criteria for refinement were used (1) VOF in between 0 and 1, and (2) a non-zero gradient of VOF. The second criterion ensures cells are not refined in regions where there is a small background value of VOF. In the cases presented in this study, the AMR plays a pivotal role: in general, by decreasing mesh spacing by 2 everywhere, computational effort increases by a factor of 8, i.e., 2 times in each spatial dimension. If the smallest cell used in the AMR mesh was to be used everywhere this resolution might be needed on a fixed mesh the resulting equivalent mesh would be far bigger. AMR reduces the computational cost by orders of magnitude maintaining the same quality of results for the interface.

\section{Geometry and Meshes}

In this paper, a generic wing mirror shape is used, which consists of half cylinder of diameter $\mathrm{D}=0.2 \mathrm{~m}$, and height $\mathrm{H}=\mathrm{D}$ ended with a quarter sphere (see Fig. 1). The same simplified geometry was used in work of [11] and [12] for an aeroacoustics study. Additionally, in this work, the influence of the presence of $3 \mathrm{~mm}$ square channel was studied, which is located $3 \mathrm{~mm}$ from to the edge of the wing mirror. The channel represents an EWM feature that might be found on a road vehicle as well as providing an additional test of the stripping models. The computational domain consists of an inlet plane placed 15D upstream, and outlet plane 30D downstream. Top and side boundaries were $15 \mathrm{D}$ from the centreline. Similarly to $[11,12]$, the inlet velocity was set to $U_{\infty}=39 \mathrm{~m} / \mathrm{s}$ (which is equivalent to a vehicle speed of $140 \mathrm{~km} / \mathrm{h}$ ) and no perturbations were applied at inlet. There is a no-slip condition on the bottom wall.
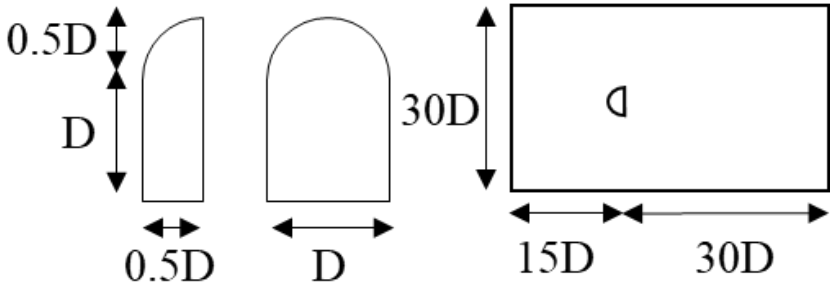

Figure 1. Wing mirror geometry: (from left to right) side view, front view, and top view with test plate dimensions.

In the Lattice Boltzmann simulations, a regular mesh (lattice) was used with refinement regions shown in Fig. $2.1 \mathrm{~mm}$ cells were used closest to the wing mirror, and the wake was refined with $4 \mathrm{~mm}$ cells. A total of $15 \mathrm{M}$ voxels were used. A fixed time-step was used with CFL number below 0.15. For the CLSVOF simulations two computational meshes were used with similar block structured mesh distribution and refinement near the wall (see Fig. 3). The first mesh of $5 \mathrm{M}$ cells was used in a single phase simulation (LES with
Smagorinsky sub-grid model) to establish a flow field around the wing mirror. This was done in the computational domain of Fig. 1 with a first cell layer thickness of 0.02 to $0.05 \mathrm{~mm}$ on the wing mirror. On the upstream side of the mirror the $y+$ was below 6.2 , with the average of 2.3. Similarly, on the back side of the mirror $y+<7.5$. On the bottom wall the $y+$ was in the range 1.5 to 306 with the highest values away from the body.

The results obtained on the first mesh were subsequently mapped to a much smaller computational domain of a base mesh of $5.6 \mathrm{M}$ cells with smaller mesh spacing (see Fig. 3), to run the multiphase simulation. The near wall thickness was larger than in single phase mesh, namely $0.25 \mathrm{~mm}$, as a lower aspect ratio of cells was required for a better description of $3 \mathrm{D}$ interface shape. This mesh was refined during the simulation in the AMR process. Two and three levels of AMR were used meaning the cells at the interface are four and eight times smaller respectively than the base mesh. The approach of mapping flow field from the bigger domain to smaller reduces the computational cost of multiphase simulation.

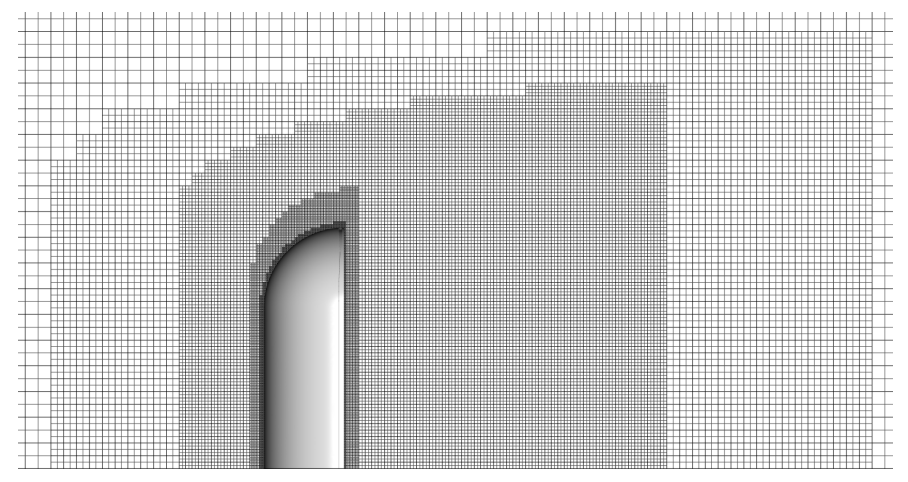

Figure 2. Mesh and refinement zones used in Lattice Boltzmann simulation.
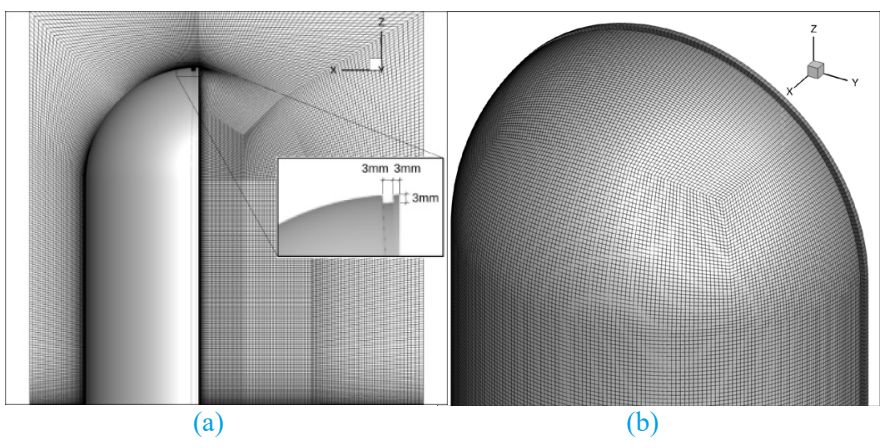

Figure 3. Base mesh used in AMR CLSVOF simulation. Note the channel located close to the edge of the mirror shown inset in (a).

\section{Flow field around wing mirror}

This particular setup was chosen to match to the work of [11] and [12] as they provide a thorough analysis of this configuration of flow studied numerically and validated against experiments with very good agreements. The main focus of [11] and [12] was to predict noise generation thus correct turbulence modelling was of high importance. In this study, the flow field around the wing mirror, especially on the upstream side, is of high concern as well, due to high aerodynamic forces exerted on the interface of liquid, causing its deformation and movement.

Page 3 of 12 
Here, the LES simulation was initialised with potential flow solution. After running for $0.1 \mathrm{~s}$ ( 20 flow through times) the statistics were extracted for the $\mathrm{t}=0.1-0.25$ (further 30 flow through times). The flow was averaged in LBM simulation for the same period of time. The mean velocity magnitude contours and streamlines are presented in Fig. 4 for the LBM simulation and in Fig. 5 for the LES simulation. In both cases the key features of the flow were captured: the large recirculation zone, which in LES case expands further downstream, two counter-rotating vortices seen from the top view at $\mathrm{z} / \mathrm{D}=0.75$, the stagnation line on the centre line of the upstream side of the mirror and horse-shoe shaped vortex upstream the body and close to the base plate. The wake is symmetric in both cases (see Figs. 4(b) and 5(b)) which indicates sufficient averaging period.

Based on the diameter, $\mathrm{D}$, the Reynolds number of this simulation is $5 \times 10^{5}$ which is close to the critical Reynolds number for both the sphere and cylinder, at which laminar-turbulent transition occurs [11]. An upstream laminar separation was observed in the experiments and from the oil film visualization of the flow in [11], an upstream separation line was located $0.15 \mathrm{D}$ from the rear edge. The wall shear stress of LES results presented in Fig. 6(a) indicates a separation at $0.1 \mathrm{D}$ which is in good agreement with both the experiments and simulations of [11]. The LES was a particularly good choice for turbulence modelling in this case, as the Detached Eddy Simulation (DES) of [11] showed a complete lack of separation on the front part of the mirror, which was explained by excessive production of turbulent viscosity, which prevents the separation occurring. Although the separation of LBM simulation is not shown directly, it can be seen in Fig. 4(b) that the wake is wider than the wing mirror diameter thus suggesting separation occurred before the end of the surface here as well. Some differences are seen in the velocity near to the separation point in Figs. 4(b) and 5(b). The largest difference is seen towards the widest part of the mirror on the cylindrical (in-board) section with the highest velocity being approximately $50 \mathrm{~m} / \mathrm{s}$ in OpenFOAM but nearer to $55 \mathrm{~m} / \mathrm{s}$ in the same location from PowerFLOWTM. However, the CLSVOF results presented later in this paper show that the film breaks down into rivulets before stripping well upstream of this point and hence this difference should not affect results greatly. The peak velocities around the end of the mirror are much closer from the two solvers. The pressure contours on the back side of the wing mirror and on the base plate are shown in Figs. 6(b)-6(d) and a very good agreement was observed with the corresponding figures of [11]. The asymmetry in the mean and RMS of pressure suggests that further averaging may be needed to obtain the true mean of these. However, as the film development and stripping are inherently transient phenomena, the mean flow field is less important than the flow being fully developed.
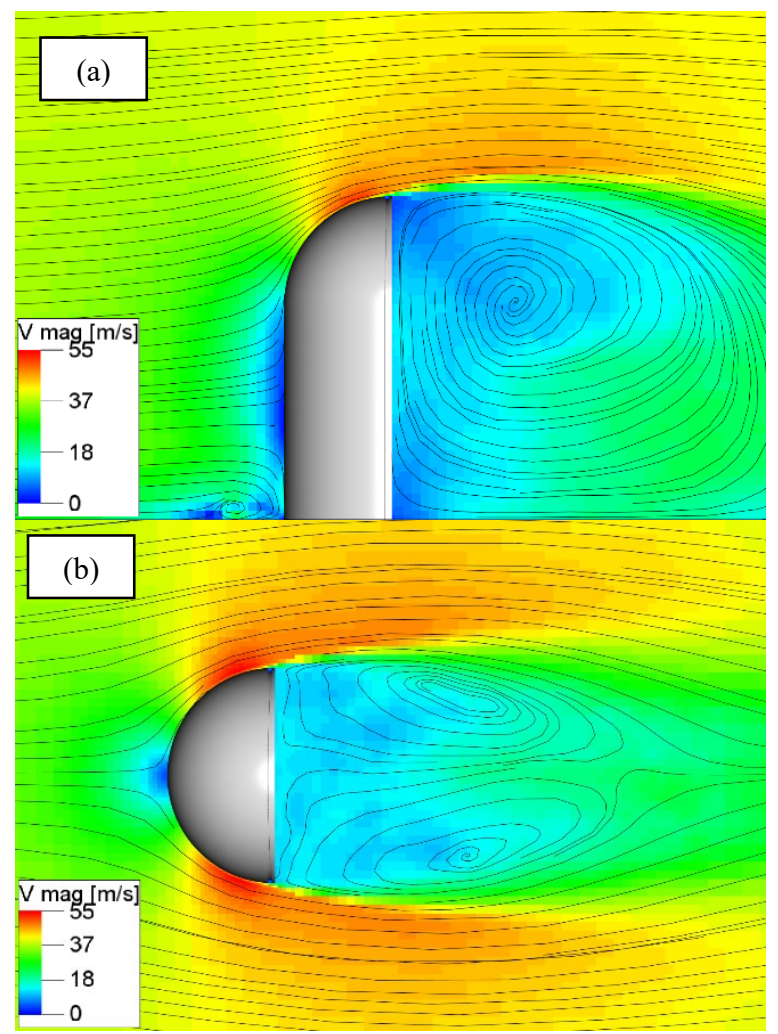

Figure 4. Flow field predicted by PowerFLOWTM, contours of velocity magnitude and streamlines, averaged over time $0.1-0.25 \mathrm{~s}$. The timescale is $\mathrm{D} / \mathrm{U}_{\infty}=0.005 \mathrm{~s}$. (a) side view $\mathrm{y}=0$, (b) top view $\mathrm{z} / \mathrm{D}=0.75$ (half the total height of the wing mirror).

In Fig. 7 an isosurface of the time mean second invariant of the velocity gradient (Q-criterion) is presented which shows the bow vortex in more details. The vortex resides on the base plate and curves around the wing mirror following the flow direction. Between the vortex and body, there is a second smaller bow vortex, which was also observed by [12] in LES simulation and in experimental flow. It was shown in [12] and references therein that coarser mesh or RANS simulations were unable to capture both of these structures. 


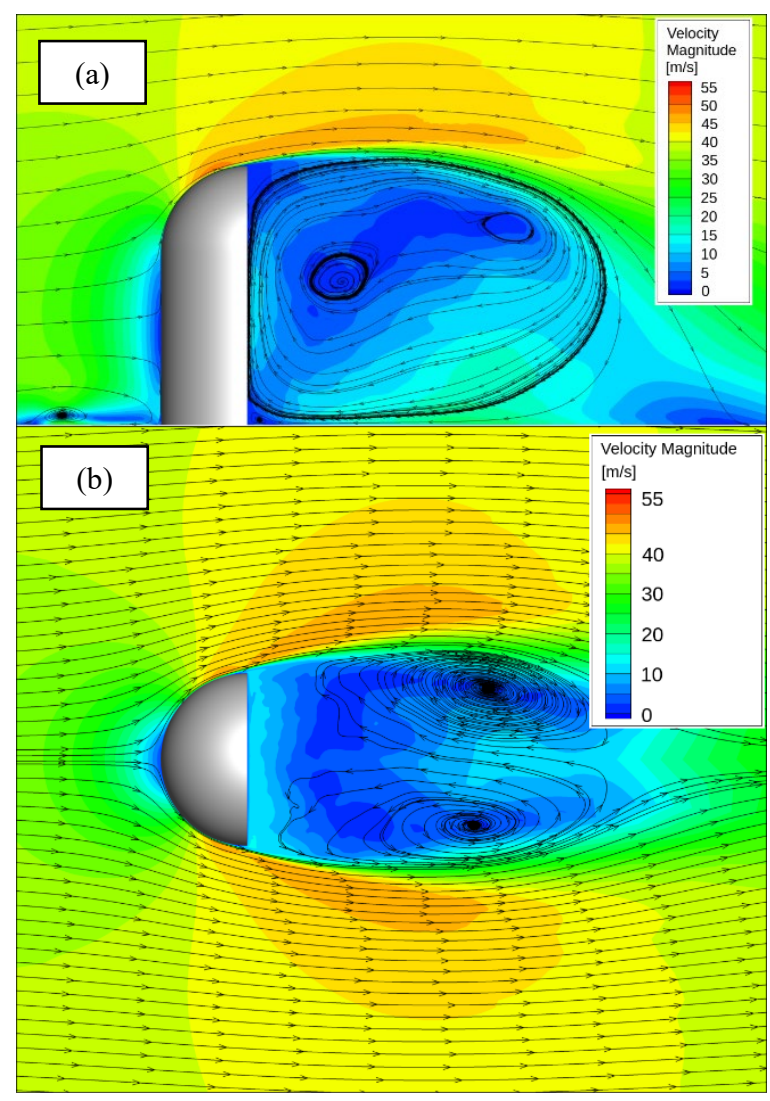

Figure 5. Flow field predicted by OpenFOAM, LES, contours of velocity magnitude and streamlines, averaged over time $0.1-0.25 \mathrm{~s}$. The timescale is $\mathrm{D} / \mathrm{U} \infty=0.005 \mathrm{~s}$. (a) side view $\mathrm{y}=0$, (b) top view $\mathrm{z} / \mathrm{D}=0.75$ (half the total height of the wing mirror).

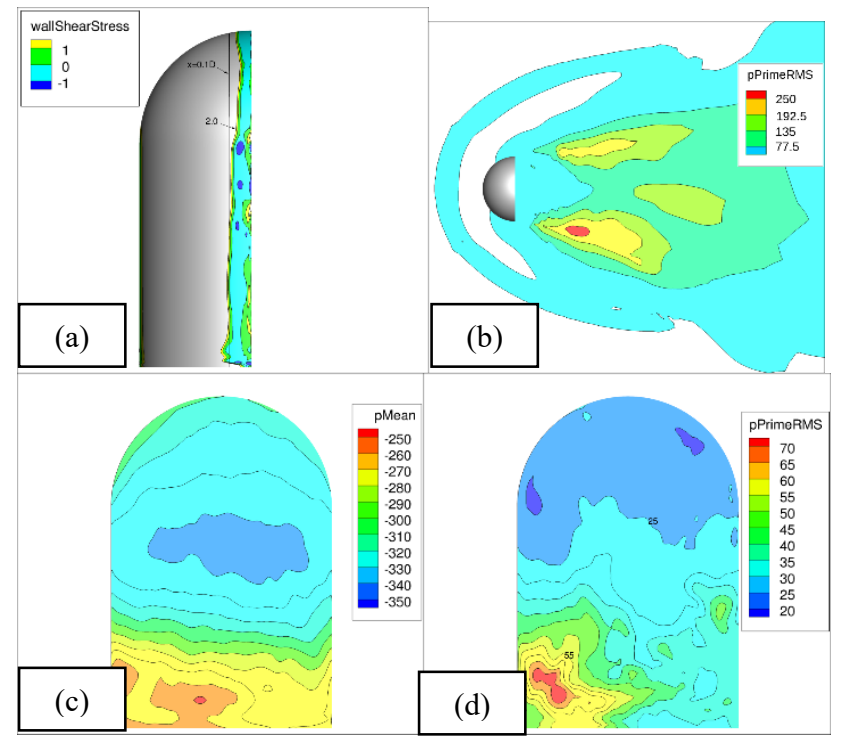

Figure 6. Flow field predicted by OpenFOAM, LES. (a) wall shear stress, (b) RMS of pressure fluctuations on the base plate, (c) mean pressure and (d) RMS of pressure fluctuations on the back of the wing mirror.

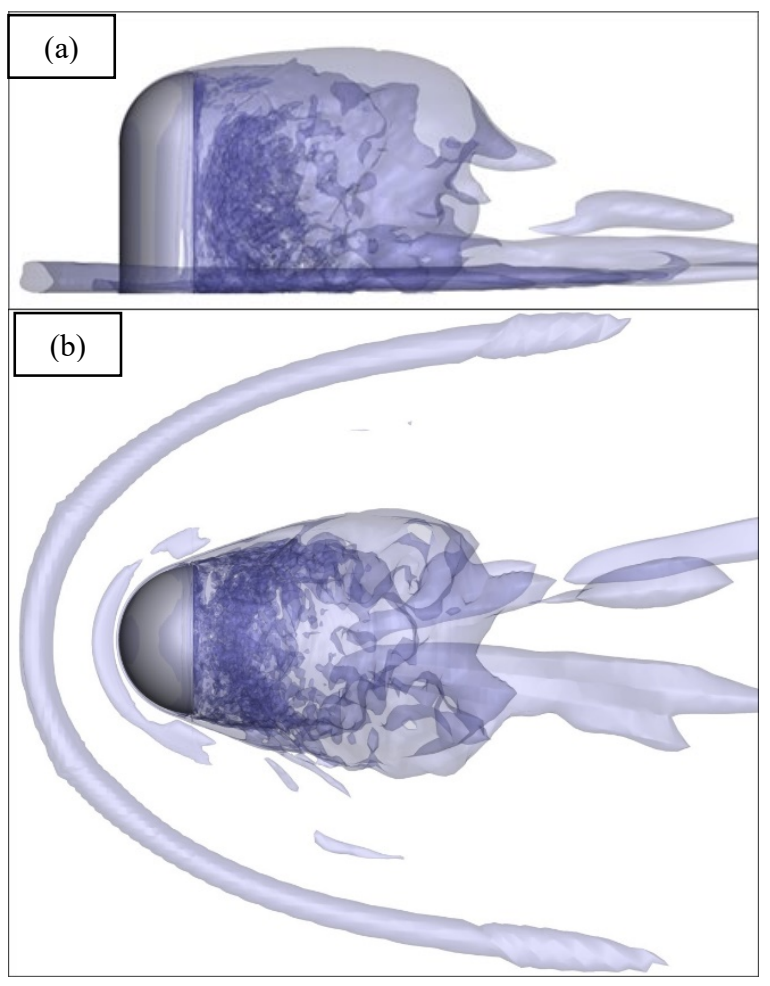

Figure 7: Flow field predicted by OpenFOAM LES, isosurface of Qcriterion equal to $10^{4} \mathrm{~s}^{-2}$

\section{Multiphase Results}

\section{CLSVOF Predictions}

The case studied in this paper is devoted to liquid flow on a solid surface and its re-entrainment into the air flow field. To avoid numerical complexity of simulating droplets impacting the surface that will eventually build up a liquid layer over time, the water is injected through a slot placed on the centerline along the body on the cylindrical and spherical parts. The width of the slot is $3 \mathrm{~mm}$ and the inlet velocity of the liquid is $0.1 \mathrm{~m} / \mathrm{s}$ which gives a flow rate of $0.000132 \mathrm{~m}^{3} / \mathrm{s}$. For comparison a heavy rain of intensity $7.6 \mathrm{~mm} / \mathrm{h}$ will give flow rate of order of $1 \times 10^{-6} \mathrm{~m}^{3} / \mathrm{s}$. For more realistic conditions (heavy rain for example) these simulations would need to be run for equivalently longer times increasing computational costs, therefore higher flow rates were used to accelerate liquid build up on the surface. The injection velocity is low enough to neglect the momentum of liquid as it builds up on the surface. The film is introduced into a fully developed single phase flow field. All times given for the multiphase results are referenced to the first introduction of the liquid through the slot. The CLSVOF simulations were run with two and three levels of adaptive refinement and no significant difference was observed in the results, giving confidence that results are mesh independent at two levels of adaptive refinement. To capture the motion of the interface a max CFL of 0.5 was used for all simulations. The simulation was run on 756 CPUs and took 90 hours to produce $0.23 \mathrm{~s}$ of simulated time.

The CLSVOF simulation shows that the liquid flow on the wing mirror can be divided into three parts: on the lower (cylindrical) part of the wing mirror the movement of liquid is influenced by gravity (in -y-axis direction) and flows downwards in a film; on the spherical 
part of the wing mirror the liquid moves in a region of high shear airflow and the influence of gravity is hardly seen (see Fig. 8). The third part, where liquid behaves differently, is where the wing mirror is in contact with the solid wall. In the flow field, the bow vortex is apparent in this location (see Fig. 7) and a rivulet flow is observed at the junction in the multiphase simulation with the film formation inhibited just above this.

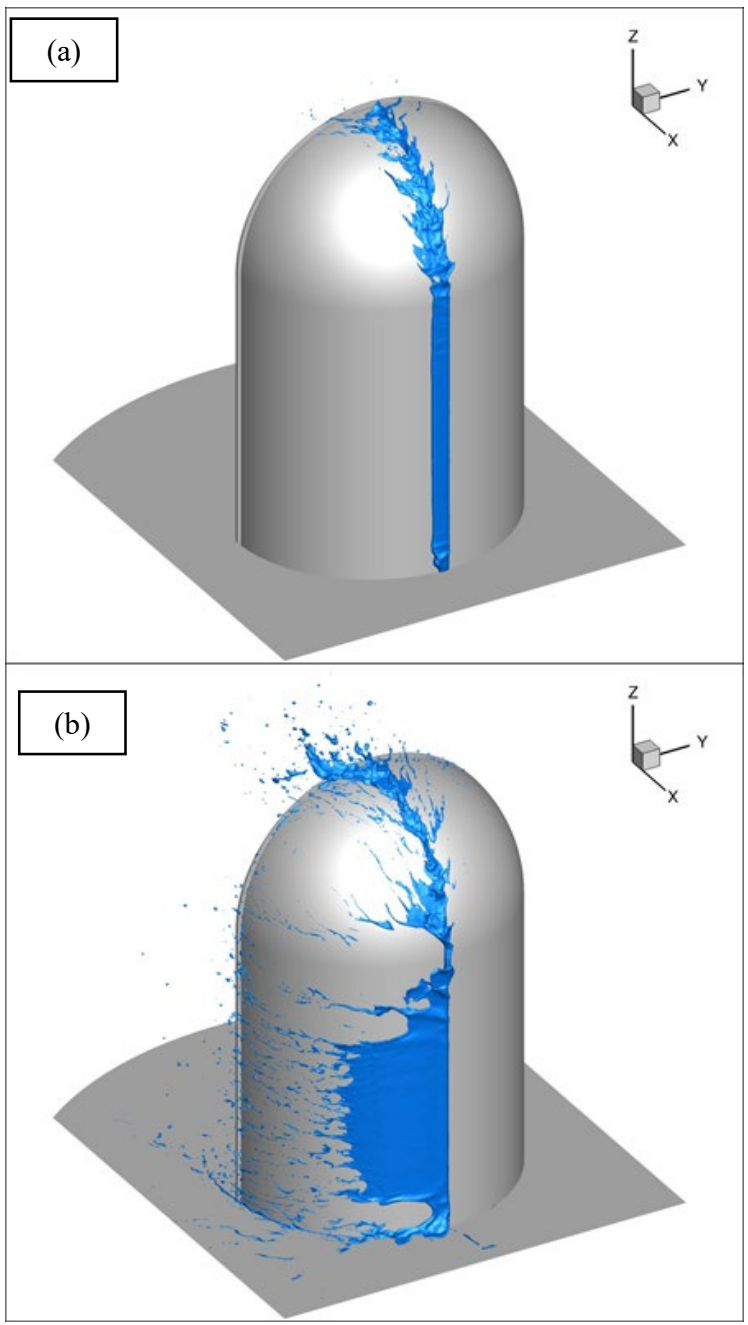

Figure 8. Results of CLSVOF simulation at (a) $t=0.042 \mathrm{~s}$ and (b) $\mathrm{t}=0.23 \mathrm{~s}$. Water surface as indicated by zero isosurface of level set field is shown.

When considering the air flow field, the stagnation line is located on the cylindrical part of the wing mirror as can be seen in the Fig. 5, and therefore, the film initiated in this region is not subjected to high aerodynamic forces. The liquid builds up on the line and eventually moves due to gravity creating a thin film of a thickness $1.5 \mathrm{~mm}$ close to the inlet which decreases in the azimuthal direction down to 0.25 mm (see Fig. 9) as the film accelerates. The shear stress in the direction normal to body surface is calculated as

$$
\tau=\mu \frac{\partial(\boldsymbol{u} \cdot \boldsymbol{t})}{\partial \boldsymbol{n}}
$$

Where $\boldsymbol{t}$ is the tangential vector to the body, $\boldsymbol{n}$ is normal to the body, $\mu$ is viscosity and $\boldsymbol{u}$ is velocity. The shear stress (see Fig. 9) increases in the azimuthal direction with air velocity increase around the wing mirror and the film, as it decreases its thickness, breaks up into rivulets, which subsequently break into droplets (see Fig. 8(b)). The droplets flow to the edge of the channel in which part of the fluid accumulates but most strips off into the wake as can be seen in Fig. $10(\mathrm{a})$.

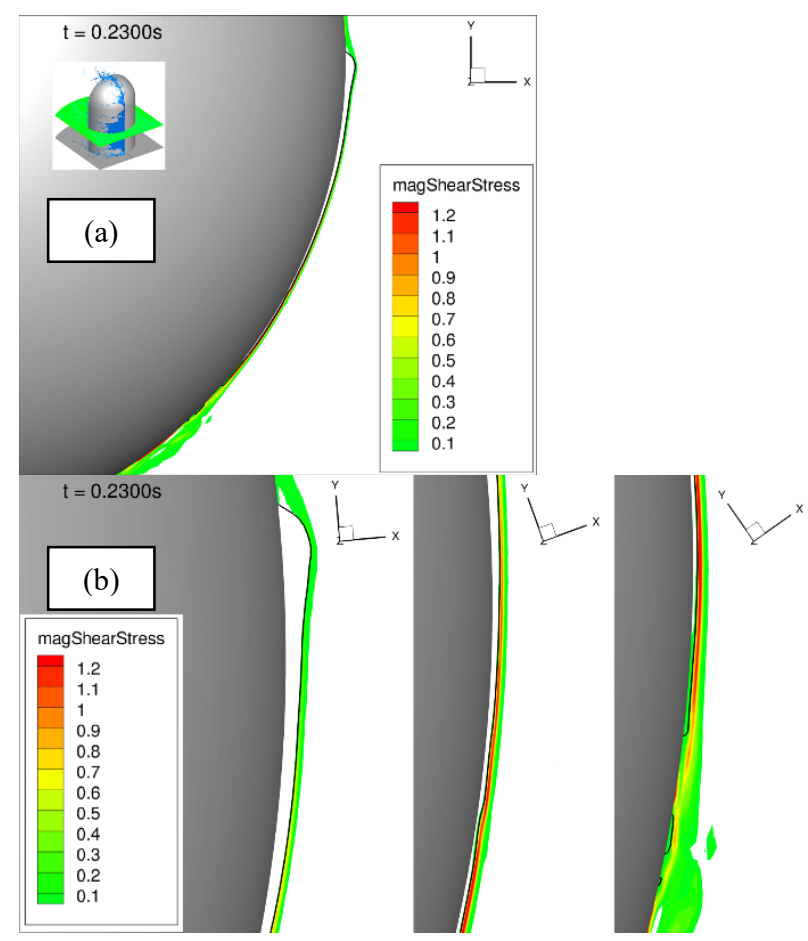

Figure 9: Results of CLSVOF simulation at $\mathrm{t}=0.23 \mathrm{~s}$. Normal shear stress and liquid surface on plane $\mathrm{z}=0.1 \mathrm{~m}$. In (b) a closer view of (a) is presented in three parts.

The behaviour of the liquid is different towards the end of the body where shear stresses in air flow field play a pivotal role. In this region the film is no longer in a stagnation region. The initially undisturbed surface of the liquid is subjected to the Kelvin-Helmholtz instability $[20,21]$, as a result, the creation of waves on the interface is observed which eventually break up and release drops into the wake (see Fig. $11)$, where the shear stress is also shown. The water re-entrains into the wake not only in the form of small droplets but also in larger structures. Not all the liquid detaches from the surface but flows in form of rivulets and droplets towards the channel. The water accumulates at the tip of the body and strips off from the edge in larger structures of liquid as can be seen in Fig. 10(b). 


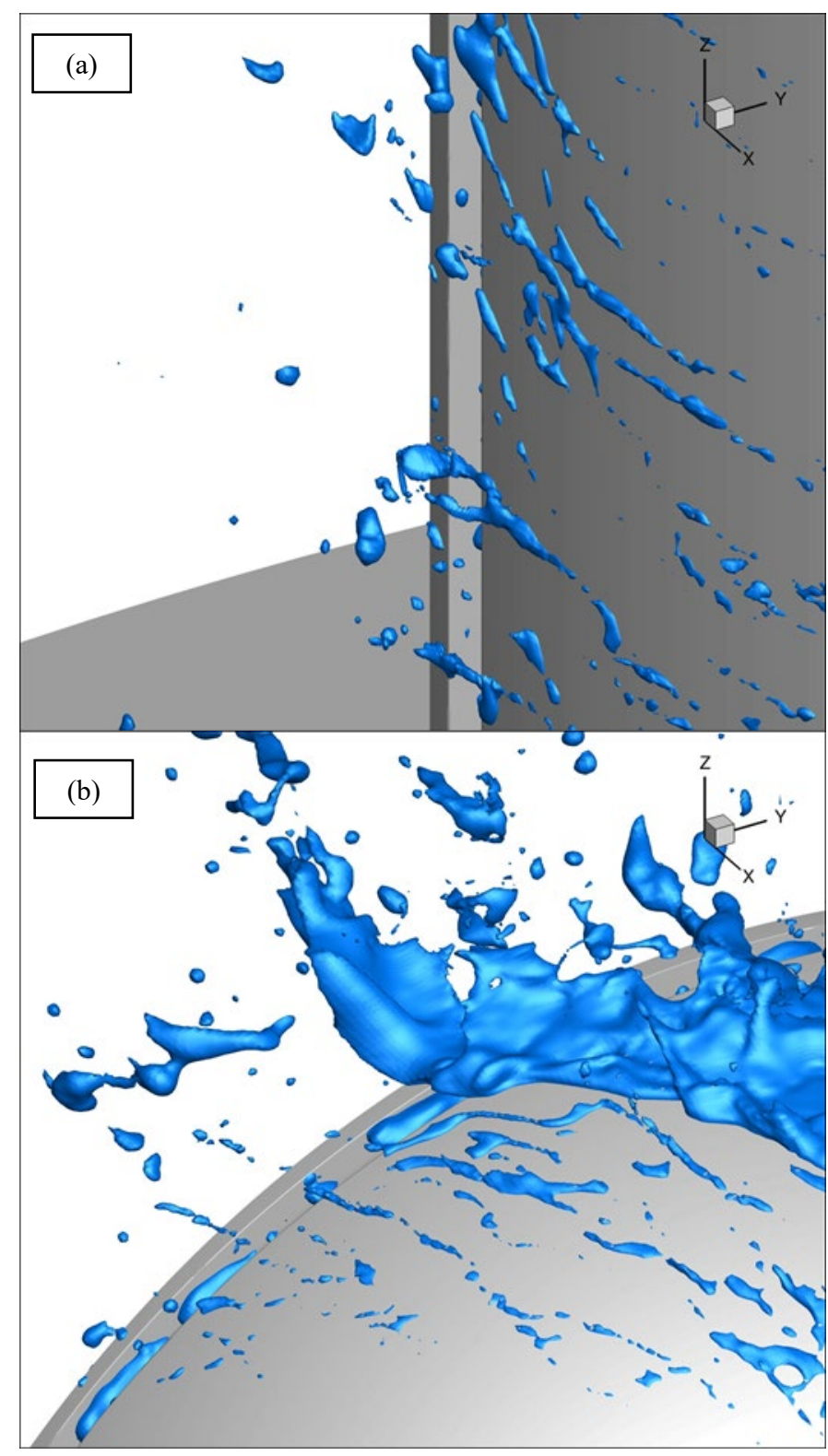

Figure 10: Closer view of results of CLSVOF simulation at $t=0.23$ s. (a) Side edge stripping, (b) top end stripping.

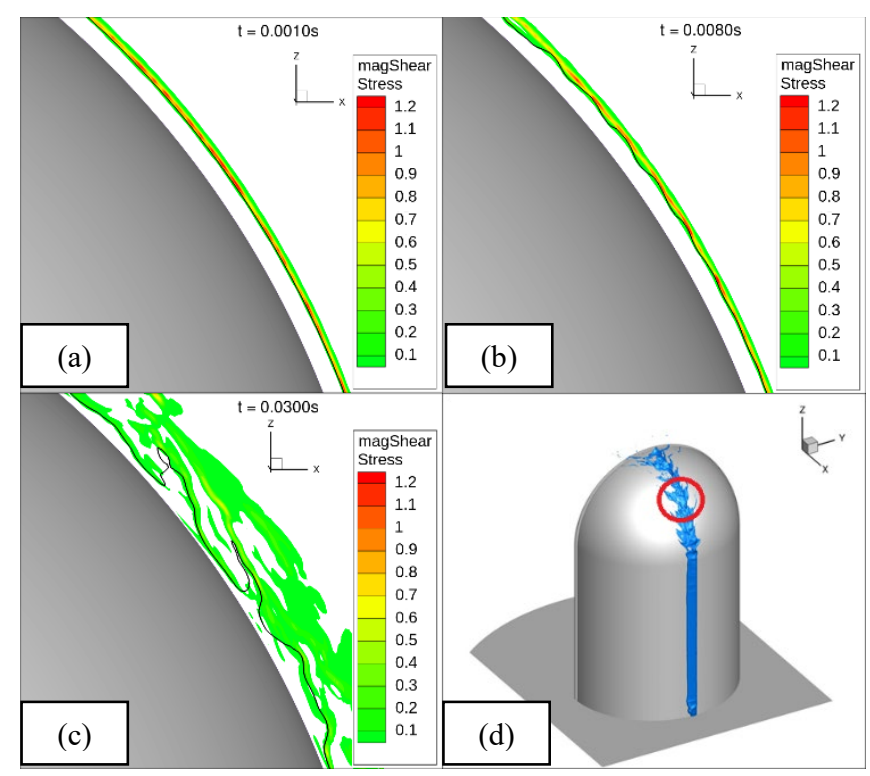

Figure 11: Results of CLSVOF simulation. Normal shear stress contours and liquid surface on plane $y=0$, for time (a) $t=0.001$, (b) 0.008 and (c) $0.03 \mathrm{~s}$. Generation of waves and breakup is observed. Location of detail shown in (d).

\section{Fluid Film Model Results}

\section{Film Height Entrainment Model}

The same configuration of the flow has been simulated with the thin film method in PowerFLOW ${ }^{\mathrm{TM}}$ for direct comparison with the highfidelity results of CLSVOF simulation. For re-entrainment, the first model considered is based on film thickness - when the thickness exceeds certain value, mass is removed from the film and particles are emitted to the gas phase. Three values were tested: $0.3,1.0$ and $2.0 \mathrm{~mm}$. The results are presented in Fig. 12 at time $\mathrm{t}=0.08 \mathrm{~s}$ and in Fig. 13 at time $\mathrm{t}=0.23 \mathrm{~s}$ for all the tested thickness thresholds. Shown are the film height contours on the surface and isosurfaces of Fluid Volume Ratio (FVR, defined as particle number density multiplied by mean particle volume) which indicates where spray is emitted. It can be seen that for $0.3 \mathrm{~mm}$ model the particles are emitted along the injector line and are advected to both sides of the wing mirror whereas in CLSVOF simulation the liquid was apparent only on the one side. Similar behaviour is observed for $1.0 \mathrm{~mm}$ model, only the $2.0 \mathrm{~mm}$ model emits particles to one side only.

As the mechanism of re-entrainment is based on liquid thickness rather than shear stress, it can be seen in Fig. 12 that the liquid is released in the end part of hemisphere region, where thickness grows due to accumulation on the edge, and from the central part of the injector line, where liquid does not yet move due to the stagnation line of the airflow. Stripping from the latter location is not seen with the CLSVOF results. When comparing liquid film on the surface at the later time of $\mathrm{t}=0.23 \mathrm{~s}$ (Fig. 13), the $1.0 \mathrm{~mm}$ and $2.0 \mathrm{~mm}$ models both predicted a film coverage with similar extent to the CLSVOF results but only on the cylindrical part of the wing mirror. In the hemispherical region at the end of the wing mirror both $1.0 \mathrm{~mm}$ and $2.0 \mathrm{~mm}$ results incorrectly predict the film to extend over the surface of the geometry rather than being stripped off quickly, as in the CLSVOF results. Whereas in the $0.3 \mathrm{~mm}$ model the film behaves correctly in the end region but not in the cylindrical part. Hence it is clear that no threshold height is capable of giving the correct stripping behaviour in all regions. 
Fig. 14 shows the rear side of the wing mirror for all three models at $\mathrm{t}=0.23 \mathrm{~s}$. It can be seen that in models using 1.0 and $2.0 \mathrm{~mm}$ a significant amount of liquid flows to the back side, contrary to CLSVOF simulation in which the rear remains dry. This is caused by the lack of any edge stripping mechanism in the thin film method which should reduce the amount of liquid that flows around the edge. Any particles that strip off the edge are due to the increase of the film thickness at the edge. It can be also seen that in $2.0 \mathrm{~mm}$ model liquid accumulates in the channel contrary to what was observed in the CLSVOF simulation. This is mainly caused by strongly 3D effects when liquid flows around complex geometries which cannot be correctly predicted by the thin film method without additional empirical modelling.

\section{Kelvin-Helmholtz Entrainment Model}

An alternative to the fixed thickness threshold model is a model based on the Kelvin-Helmholtz (K-H) instability theory, as explained in [20]. In the K-H re-entrainment model, the most unstable wavelength is calculated:

$$
\lambda(\mathbf{x}, t)=\frac{2 \pi \sigma}{\sqrt{\frac{1}{9} \rho_{a}^{2} U^{4}+\rho_{l} \mathbf{g} \cdot \mathbf{n}}+\frac{1}{3} \rho_{a} U^{2}}
$$

Where $\mathbf{x}$ is position at the surface, $\sigma$ is surface tension, $U=U(\boldsymbol{x}, t)$ is slip velocity, $\rho_{a}$ and $\rho_{l}$ are air and liquid densities, respectively, $\mathbf{n}$ is normal to the surface and $\mathbf{g}$ is gravity. The critical thickness for reentrainment is now calculated as:

$$
h_{c}(x, t)=\frac{\lambda(\mathbf{x}, t)}{2 \pi}
$$

To assess the suitability of this model it has been tested by postprocessing the results from the film model using the fixed threshold stripping model to see where the K-H model would be active if the model had been applied in the simulation. As this is done as a postprocessing step the model can only show where re-entrainment could take place if a film is present at that point. Therefore, the results from the film height entrainment model using a threshold of $2 \mathrm{~mm}$ are used as this gave the greatest extent of film over the surface. The results using the $2 \mathrm{~mm}$ model, are shown in Figure 15 at $\mathrm{t}=0.04$ and $0.23 \mathrm{~s}$. It can be seen at the earlier time that the $\mathrm{K}-\mathrm{H}$ model predicts reentrainment mostly on the centre line at the end part of the wing mirror which is in agreement with the CLSVOF simulation. A difference to the previous case can be seen at $t=0.04$ where K-H model predicts re-entrainment in the top part of the body but also in the bottom part close to the base plate. The CLSVOF simulation in the latter location shows an instability as well caused by the bow vortex (see Fig. 7). A particularly good agreement with the CLSVOF simulation is seen at the later time of $t=0.23 \mathrm{~s}$ in Fig. 15(d) where re-entrainment is predicted in the region where, in CLSVOF, the film breaks to rivulets on the cylindrical part (see. Fig. 8(b)), as well as the centre line region at the end of the mirror.

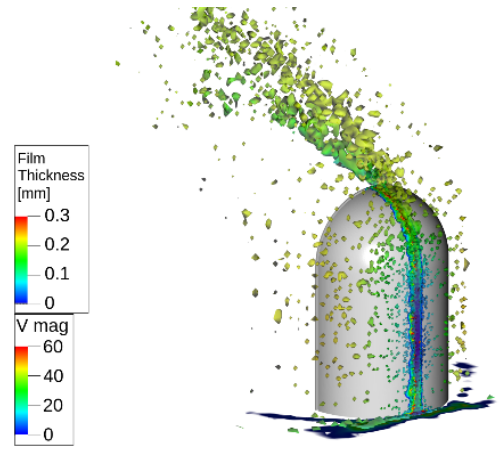

(a) re-entrainment $0.3 \mathrm{~mm}$

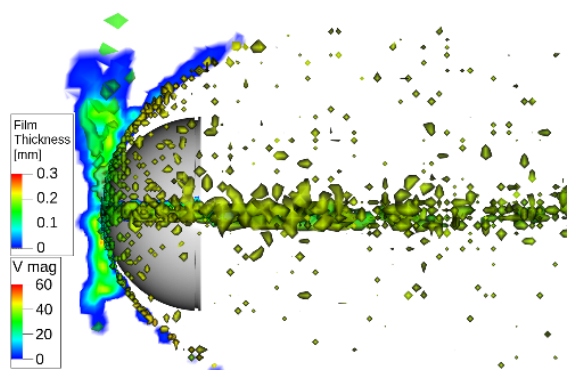

(d) re-entrainment $0.3 \mathrm{~mm}$

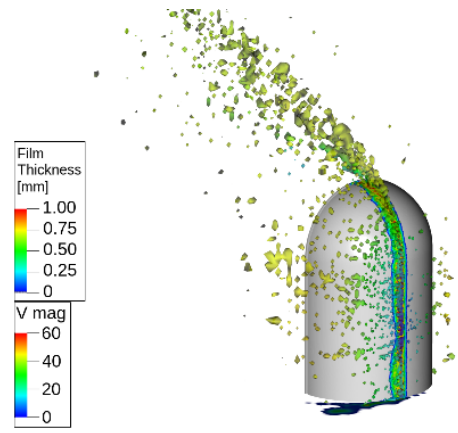

(b) re-entrainment $1 \mathrm{~mm}$

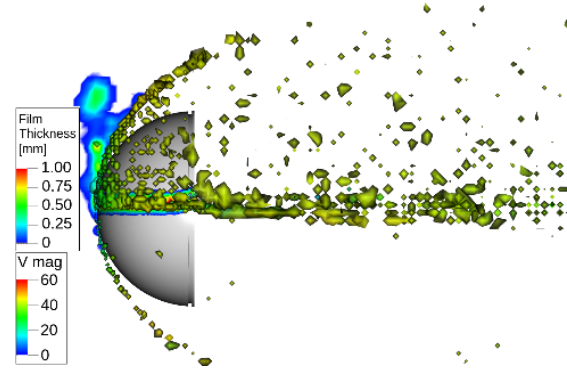

(e) re-entrainment $1 \mathrm{~mm}$

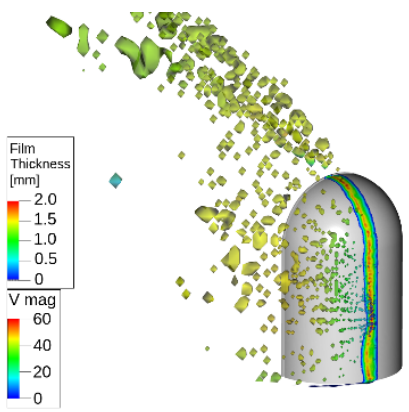

(c) re-entrainment $2 \mathrm{~mm}$

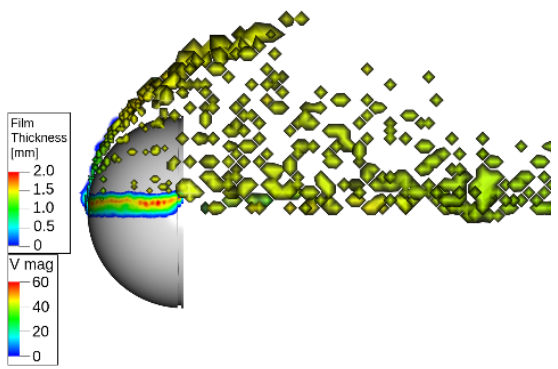

(f) re-entrainment $2 \mathrm{~mm}$

Figure 12: Results of LBM simulation at $t=0.08 \mathrm{~s}$ with thin film model and film height entrainment model with different re-entrainment thresholds. Film thickness and fluid volume ratio (FVR) isovalue of 0.001 are presented. $F V R=$ particle number density $\mathrm{x}$ mean particle volume. 


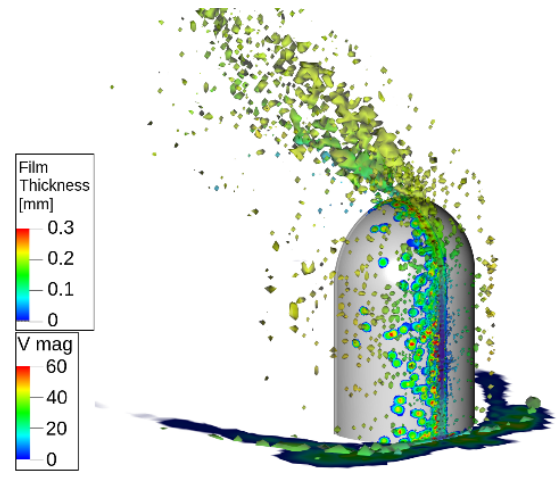

(a) re-entrainment $0.3 \mathrm{~mm}$
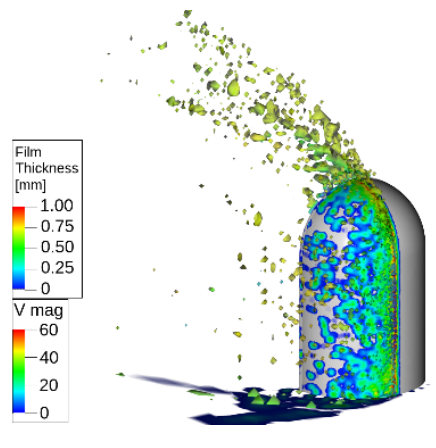

(b) re-entrainment $1 \mathrm{~mm}$

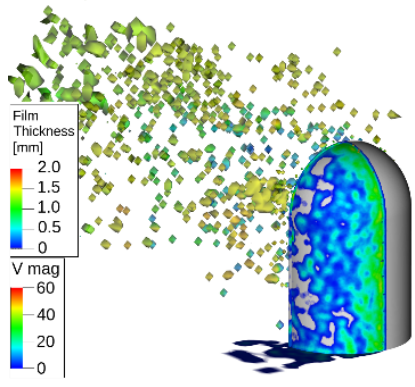

(c) re-entrainment $2 \mathrm{~mm}$

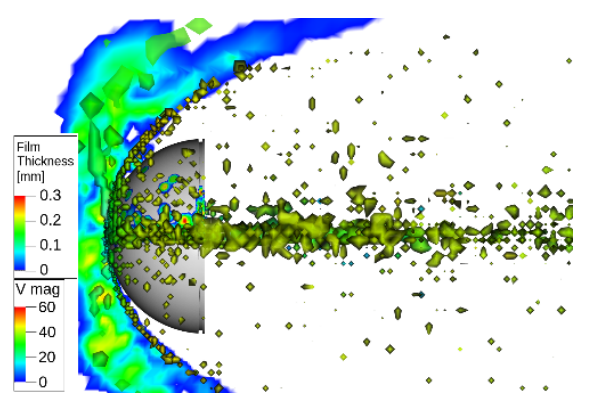

(d) re-entrainment $0.3 \mathrm{~mm}$

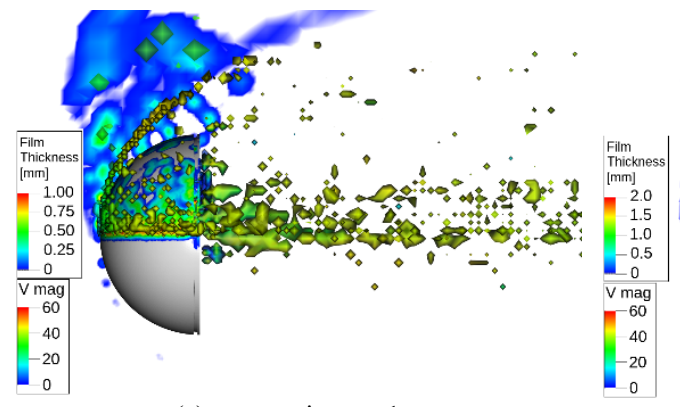

(e) re-entrainment $1 \mathrm{~mm}$

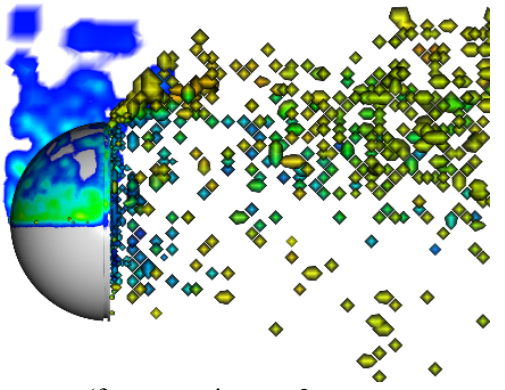

(f) re-entrainment $2 \mathrm{~mm}$

Figure 13: Results of LBM simulation at $t=0.23 \mathrm{~s}$ with thin film model and film height entrainment model with different re-entrainment thresholds. Film thickness and fluid volume ratio (FVR) isovalue of 0.001 are presented. $\mathrm{FVR}=$ particle number density $\mathrm{x}$ mean particle volume.

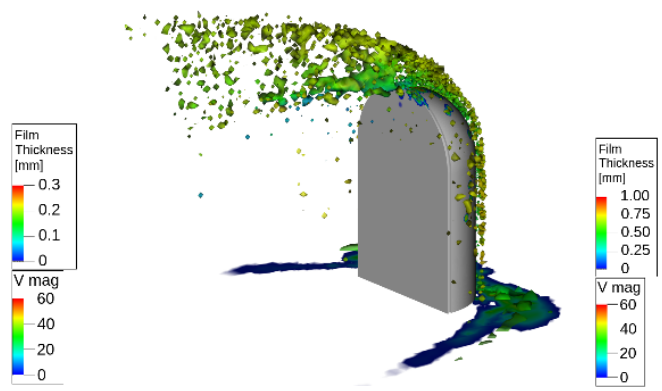

(a) re-entrainment $0.3 \mathrm{~mm}$

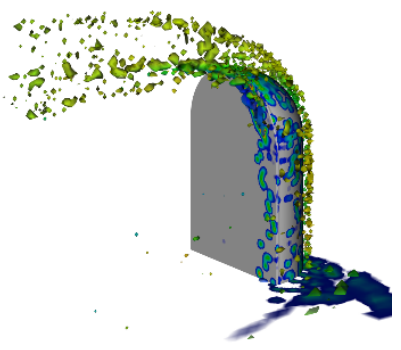

(b) re-entrainment $1 \mathrm{~mm}$

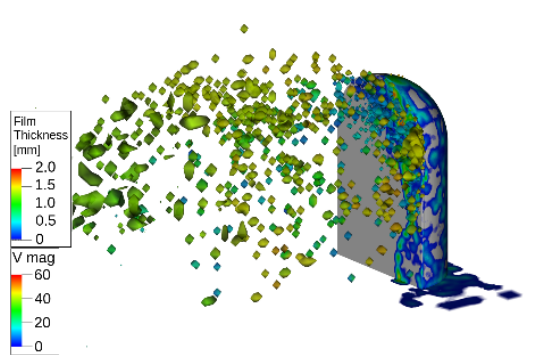

(c) re-entrainment $2 \mathrm{~mm}$

Figure 14: Results of LBM simulation at $t=0.23 \mathrm{~s}$ with thin film model and film height entrainment model with different re-entrainment thresholds. View of the back side of the wing mirror. Film thickness and fluid volume ratio (FVR) isovalue of 0.001 are presented. FVR $=$ particle number density * mean particle volume. 


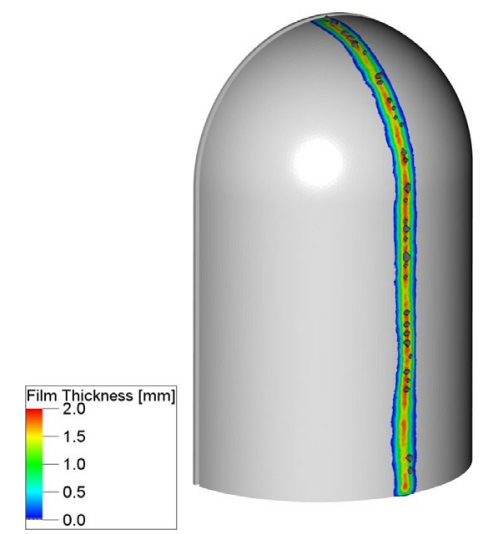

(a) $t=0.04 \mathrm{~s}$

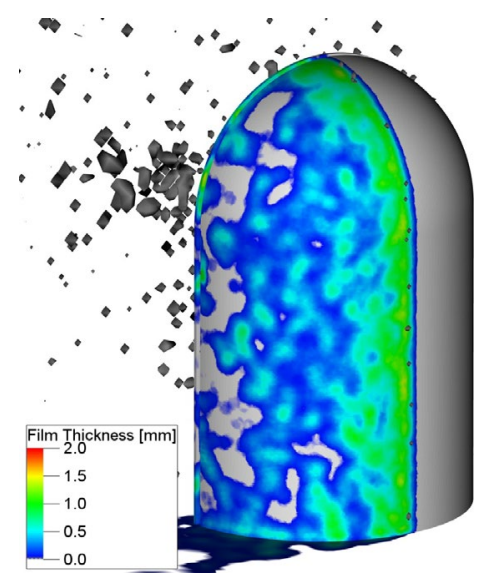

(c) $t=0.23 \mathrm{~s}$

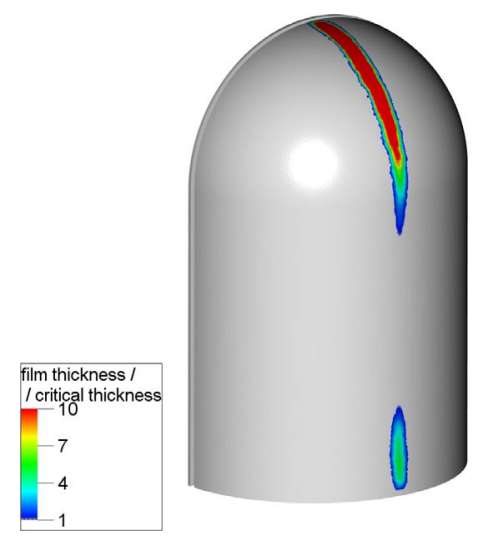

(b) $t=0.04 \mathrm{~s}$

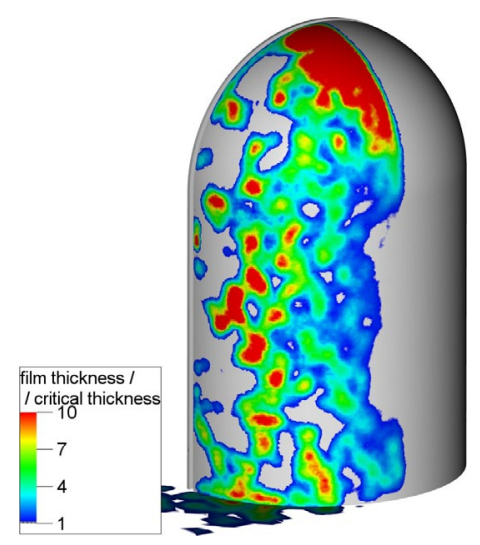

(d) $t=0.23 \mathrm{~s}$

Figure 15: Results of LBM simulation with $2.0 \mathrm{~mm}$ model at (top) $t=0.04 \mathrm{~s}$ and (bottom) $t=0.23 \mathrm{~s}$. (left) Shows film thickness and FVR, (right) K-H reentrainment model critical thickness divided by actual thickness (visible values only above one).

The validation of the K-H model gives very good agreement with the film predicted to be stripped in regions which correspond to those in the CLSVOF simulation. However, it should be noted that this is only based on post-processing film thickness data produced using the fixed thickness models. When applying the K-H model in the simulation more differences to the fixed thickness models are expected and better agreement should be achieved with the CLSVOF simulation in terms of the surface flow and the stripping. This should be, for example, apparent in the top part of the wing mirror where the CLSVOF simulation shows strong stripping and almost no liquid flows to the sides (Fig. 8(b)). In Fig. 15(c) there is a significant amount of liquid film to the side of the top part of the mirror which will be reduced by the intensive stripping occurring at the centre line. Similarly, the stripping predicted close to the base plate in Fig. 15(b) would prevent the film propagating round the mirror in this region; potentially improving the agreement with the CLSVOF results. The $\mathrm{K}-\mathrm{H}$ model does not directly model the stripping due to passing the film over a sharp edge. The benchmark results from the CLSVOF solver show that the film strips before it reaches the edge. As such the results in Fig 15(d), which show that the K-H based model predicts stripping prior to the edge, are correct in this case. However, in other cases films will reach a sharp edge and the K-H based model alone may not give the correct behaviour. It is likely that a further 'edge stripping criterion' would need to be added to the film model. A possible future extension of this work would be to use the CLSVOF solver to generate benchmark cases for other water management scenarios.

\section{Fluid Film Results with PowerFLOW'M 6-2019}

The third entrainment model tested was implemented in PowerFLOW ${ }^{\text {TM }}$ 6-2019 and hence, unlike the K-H model, is part of the simulation rather than a post-processing step. A new reentrainment model was introduced into the solver which triggers reentrainment based on the local film velocity, surface curvature and body force. The new model does not require the user to specify a fixed re-entrainment length as the local re-entrainment length is calculated by the algorithm. The results generated with this new code are shown in Figure 16. Comparing these results with the K-H reentrainment model critical thickness shown in Figure 15, and the CLSVOF results in Figure 8, the locations where re-entrainment occurs with the newer version of code show good agreement with the results from the high-fidelity CLSVOF simulation. Early in the simulation, at $t=0.04 \mathrm{~s}$, the water only re-entrains from the spherical part of the mirror. This is the location where re-entrainment was seen to occur in the CLSVOF results. Later, at $t=0.23 \mathrm{~s}$, the water reentrains from the side of the mirror housing as predict by CLSVOF as well as the K-H model. The new model shows improvement over the fixed length re-entrainment length model as the water does not reentrain from everywhere on the side of the mirror housing but only 
after it has flowed over the side and the film has increased speed. The new re-entrainment criteria also mean that the water is not flowing over the back edge of the mirror housing and depositing on the back face of the mirror as is shown in Figure 17.

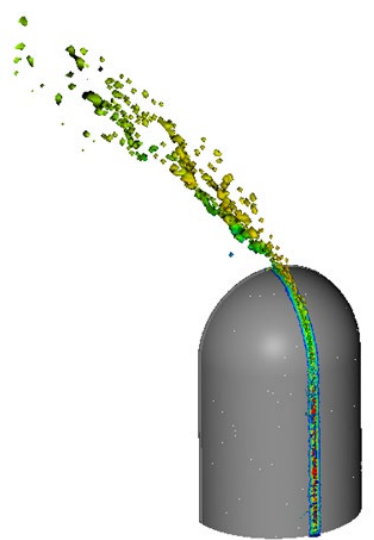

(a) $t=0.04 \mathrm{~s}$

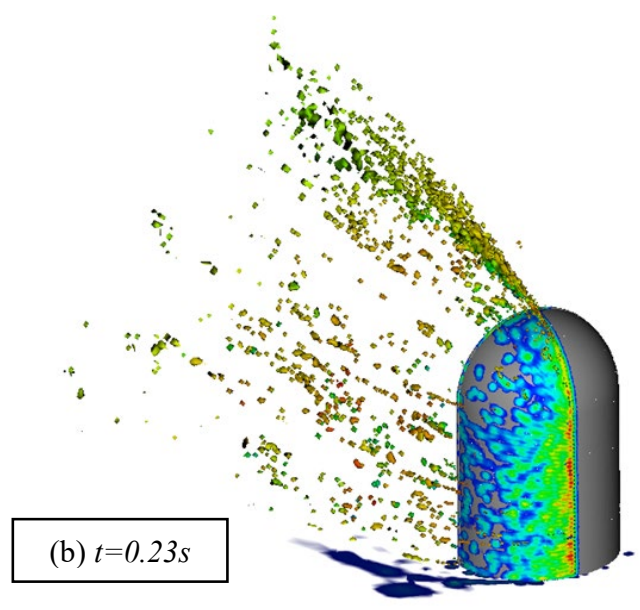

Figure 16: Results of LBM simulation with dynamic re-entrainment model. Showing film thickness and FVR.

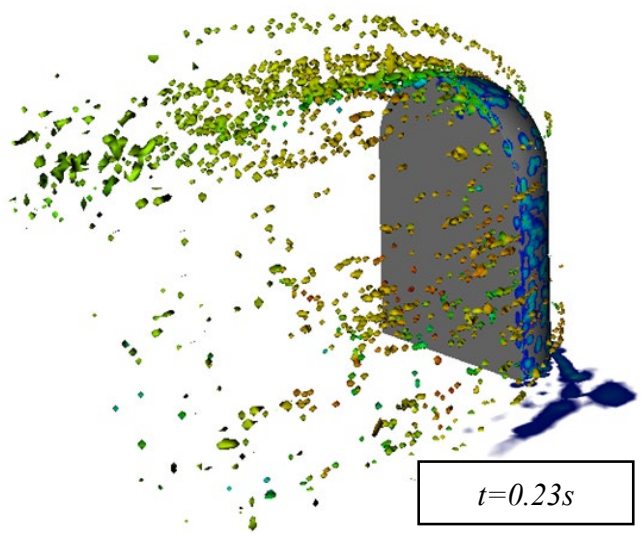

Figure 17: Results of LBM simulation with dynamic re-entrainment model. Showing film thickness and FVR. View from rear of mirror shows film does not flow onto rear surface.

\section{Conclusions}

In this paper the prediction of a water film stripping from the surface a simplified wing mirror geometry is investigated. Results using film stripping models in a fluid film model typical of practical EWM simulations are compared with a high-fidelity interface resolving simulation using a CLSVOF method. In both cases water is introduced through a slot on the surface of the mirror to allow formation of film without needing to also model the rain deposition process. Adaptive Mesh Refinement (AMR) is used in the CLSVOF simulation which allows high resolution of the process of wave formation and film stripping without the need for an unfeasible large cell count. Two and three levels of mesh refinement were found to give very similar results, showing that the CLSVOF results are mesh insensitive and can be used as a reference when comparing results using the fluid film method.

The CLSVOF simulation shows very different film behaviour on different parts of the mirror. In the central cylindrical part of the body the water forms a continuous film on the underside of the mirror due to gravity. This film thins as it accelerates, due to gravity and increasing air shear, eventually forming rivulets which strip off the edge of the mirror. At the hemispherical end of the mirror the water emerges into a region of high shear and this leads to the almost immediate stripping of the water from the surface of the mirror with virtually no film formation. Close to the junction of the mirror and base plate the film is prevented from forming by the action of a junction vortex.

In the fluid film model a stripping model based on a simple height threshold was found to be incapable of predicting all the stripping regimes with any height threshold. Using a threshold of $0.3 \mathrm{~mm}$ was found to lead to immediate stripping of the film at all points with no film development. On the other hand, using $1.0 \mathrm{~mm}$ or $2.0 \mathrm{~mm}$ led to the film forming on all parts of the mirror, including the end part where it was not seen in the CLSVOF results. A stripping model using a threshold based on the Kelvin-Helmholtz instability was found to give better agreement with CLSVOF with stripping being predicted on the centre line at the end of the mirror and towards the edge of the mirror in the central part of the body. There is also evidence to suggest that this model can predict some of the effect of the junction vortex on the film close to the base plate.

While the high-fidelity multiphase simulation method CLSVOF is not well suited for full vehicle simulations due to the computational requirements, high fidelity simulation of simplified geometries is a useful tool for developing practical engineering software. This study shows that the re-entrainment model introduced in PowerFLOW ${ }^{\text {TM }}$ 62019, exhibits similar behavior to the Kelvin-Helmholtz instability model and good agreement with CLSVOF, with stripping occurring at the end of the mirror and towards the edge of the mirror in the central part of the body. The incorporation of this model should significantly improve the results for mirror stripping and in other regions where water is removed from the surface due to curvature and shear stress considerations. This while maintaining the ability to simulate highly detailed full vehicles.

\section{References}

1. Gaylard, A., Kirwan, K. and Lockerby, D., "Surface contamination of cars: a review," Proceedings of the Institution of Mechanical Engineers, Part D: Journal of Automobile Engineering, vol. 231, no. 9, pp. 1160-1176, 2017.

Page 11 of 12 
2. Hagemeier, T., Hartmann, M. and Thévenin, D., "Practice of vehicle soiling investigations: A review" International Journal of Multiphase Flow, vol. 37, no. 8, pp. 860-875, 2011.

3. Gaylard, A. and Duncan, B., "Simulation of rear glass and body side vehicle soiling by road sprays," SAE International Journal of Passenger Cars-Mechanical Systems, vol. 4, no. 2011-01-0173, pp. 184-196, 2011.

4. Gaylard A., Fagg, M., Bannister, M., Duncan, B., Gargoloff, J. and Jilesen, J. "Modelling A-Pillar Water Overflow: Developing CFD and Experimental Methods," SAE International Journal of Passenger Cars - Mechanical Systems, vol. 5, no. 2, pp. 789800, 2012.

5. Jilesen, J., Spruss, I., Kuthada, T., Wiedemann, J. and Gaylard, A., "Advances in Modelling A-Pillar Water Overflow," in SAE Technical Paper, 2015-01-1549, 2015.

6. Kabanovs, A., Garmory, A., Passmore, M. and Gaylard, A., "Computational simulations of unsteady flow field and spray impingement on a simplified automotive geometry," Journal of Wind Engineering and Industrial Aerodynamics, vol. 171, pp. 178-195, 2017.

7. Dianat, M., Skarysz, M., Hodgson, G., Garmory, A. and Passmore, M., "Coupled Level-Set Volume of Fluid Simulations of Water Flowing Over a Simplified Drainage Channel With and Without Air Coflow," SAE Int. J. Passeng. Cars, vol. 2017, pp. $1-8,2017$.

8. Cimpeanu R. and Papageorgiou, D., "Three-dimensional high speed drop impact onto solid surfaces at arbitrary angles," International Journal of Multiphase Flow, vol. 107, pp. 192-207, 2018.

9. Dianat, M., Skarysz, M. and Garmory, A., “A Coupled Level Set and Volume of Fluid method for automotive exterior water management applications," International Journal of Multiphase Flow, vol 91, pp.19-38, 2017.

10. Skarysz, M., Garmory, A. Dianat, M., "An iterative interface reconstruction method for PLIC in general convex grids as part of a Coupled Level Set Volume of Fluid solver," Journal of Computational Physics, vol. 368, pp. 254-276, 2018.

11. Ask J. and Davidson, L., "The sub-critical flow past a generic side mirror and its impact on sound generation and propagation," in 12th AIAA/CEAS Aeroacoustics Conference (27th AIAA Aeroacoustics Conference), p. 2558, 2006.

12. De Villiers, E., "The potential of large eddy simulation for the modeling of wall bounded flows," $\mathrm{PhD}$ thesis, Imperial College of Science, Technology and Medicine, London (UK), 2006.

13. OpenFOAM v5.0, www.openfoam.org, 2017.

14. Chen, H., Kandasamy, S., Orzag, S., Shock, R., Succi, S. and Yakhot, V., "Extended Boltzmann kinetic equation for turbulent flows," Science, vol. 31, pp. 633-636, 2003.

15. Chen, S. and Doolen, D., "Lattice Boltzmann method for fluid flows," Annu. Rev. Fluid Mech., no. 30, pp. 329-364, 1998.

16. Kotapati, R., Shock, R. and Chen, H., "Lattice-Boltzmann simulations of flows over backward-facing inclined steps," International Journal of Modern Physics C, vol. 25, no. 1, 2014.

17. Mundo, C., Sommerfeld, M. and Tropea, C., "Droplet-wall collisions: experimental studies of the deformation and breakup process," International journal of multiphase flow, vol. 21, no. 2, pp. 151-173, 1995.

18. O'Rourke P. and Amsden, A., "A particle numerical model for wall film dynamics in port-injected engines," SAE transactions, pp. 2000-2013, 1996.

19. Skarysz M., and Garmory, A., "Development of an inlet boundary condition to introduce resolved droplets distribution into a multiphase simulation," in ICCFD10, Barcelona, 2018.

20. Yecko, P., Zaleski, S. and Fullana, J.-M., "Viscous modes in two-phase mixing layers," Physics of Fluids, vol. 14, no. 12, pp. 4115-4122, 2002.

21. Foucart H. and Blain, E., "Water-flow simulation on vehicle panels by taking into account the calculated aerodynamic field," tech. rep., SAE Technical Paper, 2005.

\section{Contact Information}

Maciej Skarysz

m.skarysz@lboro.ac.uk

Andrew Garmory

a.garmory@,1boro.ac.uk

Jose Escobar

jose.escobar@3ds.com

Jonathan Jilesen

jonathan.jilesen $@ 3$ ds.com

Adrian Gaylard

agaylar1@jaguarlandrover.com

\section{Acknowledgments}

M. Skarysz has been financially supported by a Jaguar Land Rover/UK Engineering and Physical Sciences Research Council (EPSRC) industrial CASE studentship ref 1689977 which is gratefully acknowledged by the authors. We acknowledge the use of Athena at HPC Midlands+, which was funded by the EPSRC on grant EP/P020232/1, for carrying out the CLSVOF simulations in this work.

Page 12 of 12 